The whole question needs consideration which hitherto it has not received in this country. Judying from the small number of cases recorded, we might be tempted to assert that they are of extreme rarity. But the fact that a single observer has met with 11 cases ocourring in about as many years at a single London hospital for chuldren suggests that such experience can hardly be unique. It is possible that, bidden away in ho-pital archives and surgeons' notebooks, other cases might be found if sought. If so we can only beg that they may be published in order to shed more light on fatalities which are as mysterious as they are tragic.

Conclusions. - 1 . That both ether and chloroform are dangerous under certian conditions at present undetermined. 2. That the symptoms suguest acid intoxication by the poisonous precursors of acetone. 3. That the origin of such poisonous bodies ( $\beta$-oxybutyric acid and the like) is in the disintegration of fat. 4 . That in practically all cases of death tollowing administration of anæsthetics advanced fatty metamorphosis is found in most organs and in the liver par exoellenoe. 5. That acid intoxication arises from such fatty metamorphosis in oryans. 6. Although prolonged administration of chloroform certainly produces general fatty metamorphosis it is incredible that such protound changes can be induced by small doses given during short operations. 7. Ether is not capable of producing similar changes, yet they are found apparently to the same extent in denths following ether as in derths after chloroform. 8 It theretore follows that such fatty metamorphosis must have existed prior to the anæethetisation. 9. The disintegration of fat into acid poisons may be due to the direct action of chloroform and ether in altering normal m+tabolism, or in some way they may favour the a.tion of bacterial toxins present in the intestines in cauring disintegration of fat. 10 . In any case the preexistence of advanced fatty changes must be presumed in order to explain fatty acid intoxication. 11. It is probable that the tatty changes in the liver are physiological and of the nat ure of it filtration rather than of degeneration. 12 If this be $\mathrm{ro}$ it is possible to understand why anæsthetics should be danyerous at one time and not at another, the element of danger being the superabundance of fat existing in the liver at the time of operation. 13. The storage of superfluous fat in the liver may in some cases be. due to the large quantities of cor-liver oil and fattening diet commonly supplied to delicate crippled and rickety children in the hope of strengthening them, coupled with want of exerci-e.

In accordance with the foregoing views the following practical couclusions may be of service in prevention of untimely deaths of children after oper.utions: 1. Before operation on even fat and apparently healthy children a careful inquiry should be made as to history of so-called "bilious attacks," which may in reality be those of "acidosis." 2. In all cases where over-fattening and want of exercise are suspected operation should be delayed if possible urtil the patient has for some days been subjected to fat-free diet. Mild purgation during this period is probably indicated. The urine should be examined for diacetic acicl and, if present, a course of alkalies, such as bicarbonate of sodium, shoull be prescribed. 3 it, should be remembered that hoth starvation and fright give rise to acetonuria. The four hours' fant usually imposed on children before operation is too long, especially when one considers that it will necessarily continue not only during the operation but for many subequent hours. Nutrient enemata should be given, after clearing the lower bowel, two hours before an operation and immediately afterwards. The effect of fright cannot be altogetber controlled but may he combated by preventing starvation. 4. The treatment of symptoms of acid intoxication following operasions should be by venesection, saline trursfusion, and by clysters of solution of bicarbonate of sodium.

1 ostsuript.-This paper was written some months ago. It is now submitted for publiuation as affording some comments on the interesting case of Delayed Chloroform Poisoning by Dr. E. W. Srott Carmichael and Dr. J. M. Beattie which appeared in THE LANCET of Augunt 12th last (p. 437), and especislly on certidin of their starements concerning it One of these strrements is: "It is interesting to note that the child vomited two or three times on the day prior to the operation." The other is in the conclusion that death from "delayed chloroform poisoning" must still be attributed to idio-y nerasp or susceptibility.

Upper Berkeley-street.

\section{THE "CONTRACTED" MUSCLES OF INFANTILE PARALYSIS.}

BY FREDERIC R. FISHER, F.R.C.S. ENG., SURGEON TO THE NATIONAL ORTHOPADIC HOSPITAL, ETC.

ANTERIOR poliomyelitis affecting that region of the cord from which are given off the roots of the lumbar and sacral nerves reduces the functional activity of the muscles of the leg and foot and in the majority of cases disturbs the balance of muscular power. From this derangement of muscular equilibrium the various conditions of paralytic talipes are developed, the particular form of distortion being determined by the relative conditions of the opposing muscles which may have been affected. In treatises on paralytic deformity the theory usually accepted as accounting for the production of talipes is that owing to the unbalanced state of the muscles the stronger have been in a condition of over-action, have overpowered their weaker opponents, and by gradually approximating their own points of attachment have become permanently shortened. This theory is certainly inaccurate in one respect, the contraction of the muscles precedes the drawing together of their points of attachment, the latter being not the cause but the result of contraction with which the shortening of the fibres is coincident. It is also extremely doubtful if a muscle will acquire from over-action a condition of permanent contraction. Many children are to be met with among the poor who, not provided with a compensating mechanical applrance, have walked for some years with the foot extended to neutralise a shortening of one lower extremity. Disease or dislocation of the hip-joint present familiar examples of such cases and amongst them contraction never results from the strain to which the extensor muscles are subjected. The causes which excite and the structural changes which constitute the condition known as "contiacted muscle" are somewhat complex in their nature; further investrgation may well be made to obtain a more reasonable explanation of these abnormal processes than is afforded by the above quoted theory.

Taking as a familiar type of paralytic deformity the case of talipes equinus illustrated by Fig. 1 an attack of anterior

FIG. 1 .

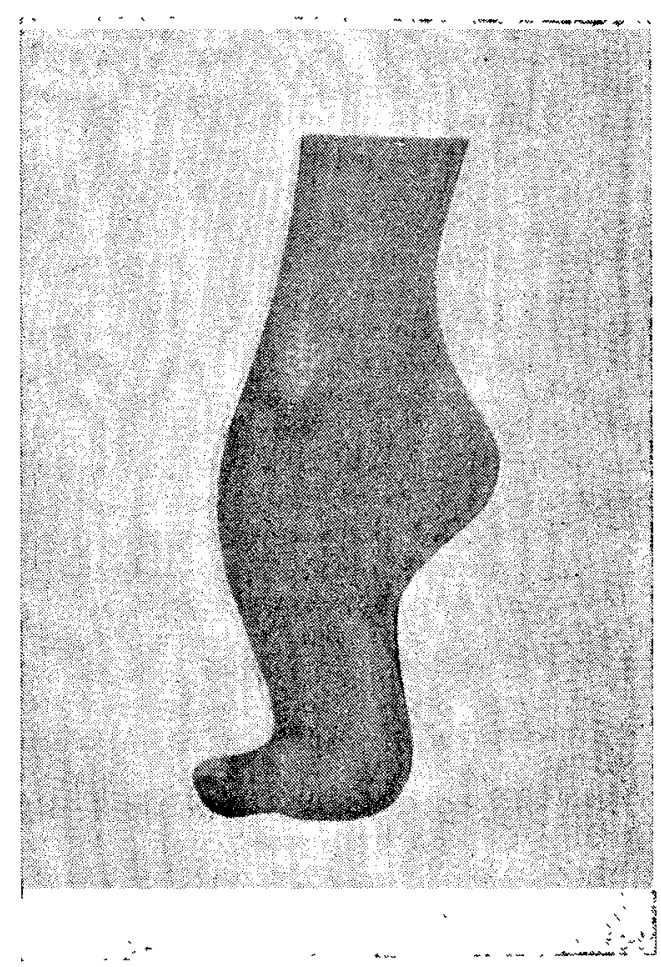

poliomyelitis has almost entirely deprived the flexor muscles of contractile power, leaving the extensors with a fair amount of activity although reduced below their normal strength. The nerve lesion in this case occurred when the subject was two years of age; the severe state of equinus exhibited was established at the age of 11 years. In the 
progress of acquirement of such detormity there will be in the first stage, when the child commences to walk after the attack of poliomyelitis, no flexion movement of the foot, nor will the heel be raised-the foot is shuffled forwards or is lifted off the ground by over-flexing the thigh. After a time, from two to three years, the extensors will increase in power, raising the heel slightly and keeping it elevated during progression. Examination at this stage will show that flexion movement at the ankle-joint is becoming limited. In conducting the examination the leg must be fully extended on the thigh and the foot flexed by placing the hand along the sole, exerting steady and gentle pressure. The foot must not be relaxed on reaching the apparent limit of movement but the pressure be continued for a minute or so, when under gradually increasing exercise of force the muscles will yield slightly and permit a further amount of flexion, probably bringing the foot nearly to a right angle with the leg. Should the child have been using the limb immodiately previous to the test it is advisable to instruct that he be brought again for examination, directing that in the interval between a night's rest and the second visit the foot should not be used in any way. At this second examination it will be found that the foot can be flexed several degrees beyond the position obtained at the first visit. Allowed to progress unchecked by treatment the heel will gradually become more elevated and subsequent examinations, say at yearly intervals, will be attended by the same conditions exhibited at the first inrestigation, modified, however, in that the yielding to continued pressure will decrease and subsidence after a night's rest will be less marked. Finally, the state of equinus illustrated becomes established, the heel is now raised to the full degree, and no yielding of the extensor muscles can be obtained by pressure, all movements both of flexion and extension are lost, and the ankle is as immobile as an ankylosed joint. A condition has now been reached in which all the muscles of the leg and foot are in like case so far as deprivation of muscular power is concerned, the feeble flexors are atrophied and elongated, and the extensors have arrived at the final stage usually attained by "contracted musales." It will be agreed that the term "contracted muscle" by no means conveys a correct idea of the muscular lesion. Contraction is a normal attribute of muscular fibre; here we have to deal with a distinct abnormality in the deprivation of the tissue of its ability to relax; "intractile muscle" better defines the state of inextensibility which is the main feature of the defect and the one upon which attention should be especially concentrated.

The case of equinus that has afforded my text exhibits only one phase of the acquirement of muscular intractility, it is the most commonly occurring type, but an almont infinite variety in the behaviour of the muscles is presented in the development of paralytic deformity. There is one constant feature which presents itself in every case of disturbed muscular balance, the stronger muscles always become intractile to some extent, but this is the limit of methodical procedure. In all other respects the muscular system, thrown out of gear by disorganisation of its motor centres, exhibits an eccentricity of conduct which affords a most perplexing puzzle for elucidation by the student. It might reasonably be expected that the greater the difference in the functional powers of opposing muscles the greater would be the degree to which the stronger would become shortened and the more severe would be the grade of deformity developed. Also when all the muscles had been seriously lowered in efficiency it might well be anticipated that those retaining superior power would, in their condition of extreme weakness, acquire but little intractility and be incapable of causing any marked degree of distortion. Yet neither of these propositions holds good in prtctice. for although examples will be met with in support of both suppositions other cases occur directly contradicting their truth. Thus, in one subject with the flexors greatly weakened and the extensors in fairly good condition the degree of intractility established is often so slight as to allow of sufficient movement for the foot to be placed at a right angle with the leg. With the flexors in like condition in another patient and the extensors so feeble as to be in scarcely better case than their opponents an extreme state of equinus may result, even to hyper-extension of the foot at the ankle-joint. I have seen examples of equinus with the foot extended to a degree which brought the articular surface of the astragalus forwards and entirely free of the ankle-joint, the displacement having been wrought by muscles so wasted that it seemed impossible they could acquire intractility. The Achilles tendon under such conditions has been as tense and the resistance of the muscular fibres equally as obstinate as in cases where the gastrocnemins and soleus muscles were fairly well preserved. Another instance of caprice is exhibited in the varying time which may elapse between the attack of poliomyelitis and the commencement of muscular shortening. It is difficult to fix the date of the first drawing up of the fibres becanse this seldom occurs under the observation of the surgeon; also, the history of the patient is not often to be obtained from a truntworthy source, and in receiving the account it is always a troublesome matter to differentiate betwixt fiction and fact. About two years may be accepted as the period of interval in the majority of instances but it may be lelayed to a later period. Certainly four or five years may eiapse, possibly even a longer time. On the other hand, the stronger muscles occasionally elect to display their superior powers even withiu a few weeks of the central attack. At 15 months of age anterior poliomyelitis caused complete paraplegia in a child who was brought to me three months later. I could excite no movement of the limbs, and giving some general directions as to the management of the case, instructed that the patient should appear again for examination after an interval of three months. At the second visit both feet were extencled on the legs to a marked position of equinus. steady pressure obtained flexion nearly to a right angle, beyond this the extensor muscles would not relax. Of course, this child had never used his legs and the conventional the ary of over action of the muscles breaks down completely when applied to such a case.

A more familiar example of unused muscles, the fibres of which have become intractile, is illustrated by the accompanying illustration ( $\mathrm{Fig} .2$ ). Both lower extremities have

Hig. 2 .

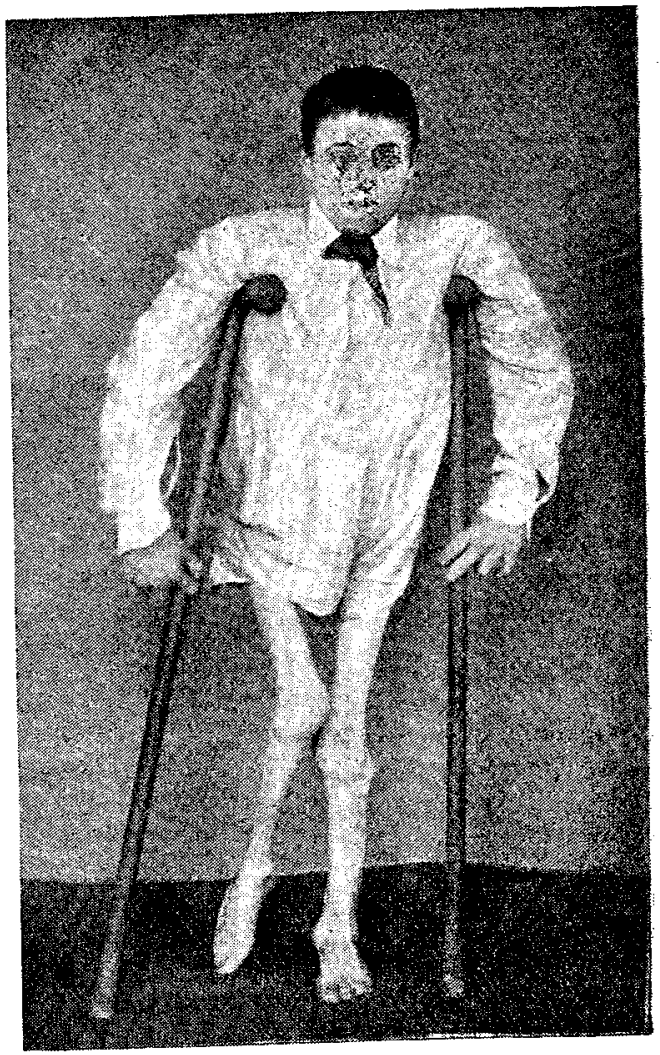

been paralysed; in the left, the better preserved, muscular balince has not been disturbed; the right limb has been so much wasted that it has never been available as a support. By the aid of crutches, and such help as the stronger extremity could afford, the patio nt has been able to progress, allowing the right limb to swing abnut as a usele s appendage to the body. In this poor shrivelled member, which might justly be described as consisting of "nothing but skin and bone," the extensors of the leg have developed. talipes equinus; the biceps femoris, in addition to producing a valgoid knee, has rotated outwards and drawn backwards the tibia upon the condyles, whilst the flexors and adductors of the thigh have united their forces and added further to 
the list of distortions. Such distortion is of twofold interest; by its existence it absolutely controverts the over-action theory and it also offers an excellent example of the development of intractility in muscles which it seems hardly possible to credit with any fibres possessing the slightest functional activity.

The uncertainty attending the period at which muscular intractility first appears, of which examples have been given, is, perhaps, surpassed by that displayed in the acquirement of permanent structural shortening of the fibres. It is impossible to fix the length of time required for the muscles to reach a condition in which their ability to relax is absolutely destroyed, indeed, it is doubtful if complete deprivation of function is ever accomplished except in cases of very long standing. Returning to the case illustrated by Fig. 1 the deformity is of a nature which I classify as tarsomalleolar equinus; one half of the distortion is due to displacement of the foot at the ankle joint, giving the malleolar character to the deformity, while the front of the foot is

FIG. 3

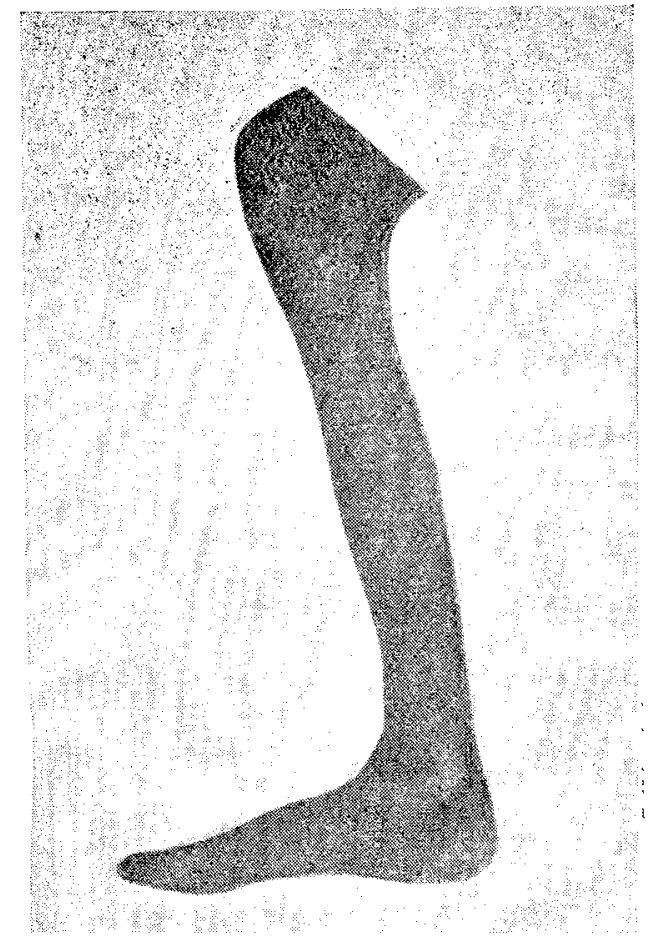

depressed upon the os calcis and astragalus, which constitute the tarsal moiety of the defect. It was necessary in conducting the treatment to reduce the displacement at the tarsal joints, astragalo-scaphoid and calcrneo-cuboid, before eorrecting the position of the foot upon the leg. This first stage of treatment occupied about seren weeks and when completed it was found that the leg could be flexed over the foot to the degree shown in Fig. 3. In this subject the muscular fibres bad been steadily contracting for a period of about seven years, the foot was reported to have been in the advanced state of deformity for two or three years and, as before stated, was rigidly fixed in its extended position on the leg. After seven weeks of absolute rest the gastrocnemius and soleus muscles had relaxed to a degree which gave a sufficient amount of flexion at the ankle-joint for all ordinary purposes. This is certainly an exceptional instance; it is seldom that spontaneous relaxation of the fibres under conditions of repose of the muscles occurs to such a degree in so short a period of time. There is, howere : in like circumstances always some decrease of intractility, a fact of supreme importance in directing the treatment of the abnormality and one which can be more conveniently discussed when the methods of treatment are under consideration.

In theorising on the development of paralytic talipes writers have hitherto overlooked the point that muscular intractility is as distinctly a loss of natural function as is muscular purdlysis: in the one case ability to relax is reduced and in the other power of contraction is lowered. In both cases the defect varies in degree from a slight aberra tion which is scarcely appreciable to advanced structural change which completely destroys the normal attribute of the muscular fibres. We know that the loss of contractile power is due to, and is in direct ratio to, the degree of destruction of the motor cells in the anterior cornua; it is possible that from the loss of a controlling influence naturally exercised by the same central structures arises the development of intractility. In the act of walking the extensors and flexors of the feet are alternately contracting and relaxing-the one set of muscles relax as the opposing set contract. If it can be admitted that these movements when once started by impulse from the cerebral centres are automatically maintained by the motor cells of the anterior cornua then it seems to me that the theory I have suggested is not only possibly but even probably correct. It could be assumed that when contraction of the muscles necessary to prodnce the required movement of the foot has been completed the fibres do not fully relax before a second contraction has commenced. The overlapping of the two actions of the muscle would result in the fibres never being completely relaxed. during the whole time that walking was in progress and it may well be supposed that when at rest some period of time would be required for them to become slack. This suggestion is supported by the different degrees of intractility which, as previously noted, are found on examining a limb immediately after it has been in use and again after a night's rest, the muscle being more extensile on the latter occasion. In the early stage of using the limb, after the attack of poliomyelitis, a night's rest will suffice to allow the subsidence of such intractility as may be acquired during the day, but as time passes the relaxing process will not be finished and the subject will start walking on the following day with the muscle still slightly shortened. Some increase of the defect will be promoted day after day, an addition too trifling to be detected by examination at short intervals of time, but sufficient by gradual accumulation during months and years to give rise to a severe grade of deformity. It may be objected that if this incomplete action accompanies the use of the stronger it should also attend upon the use of the weaker muscles in cases where the balance is but slightly disturbed and where a fair amount of power remains to all the muscles of the limb. Under such conditions the weaker muscles will never be fully contracted, even to the degree to which they are still capable of performing that function. Just as from disorganisation of the motor centres the relaxing of the better preserved muscles is not com pleted, so from the same lack of proper control the fibres of the weaker opponents are never brought to a state of full contraction. Moreover, in the variety of disturbance characterised by a moderate lessening of power of the extensors and flexors, with balance against the latter, the more active extensors will first develop a degree of intractility sufficient to limit flexion of the foot beyond a right angle and having reached this stage no further shortening of their fibres takes place. Thereupon the flexor muscles will come into fuller play and themselves acquire some degree of intractility, the result being that the two conditions-" "right-angled contraction of the tendo Achillis" and "claw-shaped toes"-which are frequently met with apart, will in these circumstances be combined in the same foot. Another incident bearing upon this point is worthy of notice-it is possible by treatment to reverse the relative conditions of opposing muscles, to set the balance against those which had been the better preserved; the result then is that the muscles which were originally the weaker acquire intractility.

In endeavouring to clear the obscurity surrounding the various changes consequent upon poliomyelitis the most difficult task is to throw light upon those cases in which the muscles, apparently deprived of contractile tissues, are able to generate very evident distortion. There must be in such a case as that illustrated by Fig. 2 some remnant of contractile tissue existing after the widespread destruction, so it may be concluded that absolute annihilation of muscular function is seldom completed. Voluntary movement can hardly account for the phenomena nor does it seem possible that reflex action can be existent. The only solution that can suggest is that some feeble stimulus may be issued from the central stations of the cord, erratic messages from a directing centre which has been thrown into a state of hopeleis confusion. Be this as it may, the development of paralytic talipes probably depends entirely upon neuropathic influence ; the muscles imperfectly stimulated are sluggishly responsive, both in the act of relaxing and of contracting.

The treatment of intractile muscles. - The ill-effects resulting from anterior poliomyelitis being chiefy due to the balance of muscular power having been disturbed treatment 
must be directed to regulate as far as possible the action of the affected muscles. Obtaining control over the more active muscles usually receives less attention than does the strengthening of the weaker opponents; this is mistaken policy, for the development of intractility in the former must offer resistance to and limit the action of the latter. The proposal to adopt any measures which will tend to lower the power of the stronger muscles is often objected to; it is argued that by such practice an already weakened limb is still further reduced in strength. That this reasoning is false may be proved by referring again to the case illustrated by Fig. 1 ; in such a case the flexors of the foot have no possible chance of regaining efficiency during the time that they are being overpowered by the more vigorous extensors. The want of a check upon the stronger muscles in such circumstances contributes to the weakening of the leg and causes the very result which the adoption of restraining measures is ignorantly accused of producing. Too much importance is also given to the reduction of such deformity as may have been developed by the dominance of the better preserved muscles, the deformity being so much in evidence that it appropriates an undue share of attention. As a rule there is very little difficulty about restoring shape to a foot affected with paralytic talipes the trouble is so to deal with the muscles that they may be able, the strong and the weak, to take up their work on fairly even terms after the re shaping of the foot has been accomplished. It cannot be too strongly insisted upon that with a case of disturbed muscular action, no matter what the grade of paralysis may be, the chief object of treatment should be to redress the balance of power. If this can be done by raising the weaker muscles to the standard of the stronger so much the better; failing this the stronger muscles must be reduced to the level of the more feeble. There may be met with one among 100 cases in which the disparity of power between sets of muscles is so marked that any interference with the strong would be inadvisable, but such an exceptional instance may be disregarded in a general review of the treatment of paralytic deformity.

In the early state of infantile paralysis, when it is seen that one set of muscles is in better condition than the opponents, much may be done to prevent the former from becoming intractile. Such movements of the foot upon the leg as will bring the stronger muscles to a full degree of normal relaxation should be practised regularly, "passive exercises" are valueless, active use of the muscles is imperative, and with very young children it requires some little ingenuity to get what is wanted in this respect. A good deal can be accomplished towards the desired end because the victims of the disease are, almost without exception, children of quick intelligence-they are excellent little patients who readily respond to the instructions they receive. Should some intractility of the fibres be already established and the full degree of relaxation not be obtainable exercises are again to be employed but no forcible extension of the fibres must be attempted. When the shortening stage has commenced anything in the way of force is immediately resisted, therefore the movements must be practised slowly and steadily, endeavouring, as it were, to coax the fibres to a state of submission. Gentle handling, previously described as very necessary when examining a case of paralytic talipes, must be most carefully observed in conducting the treatment, violent measures will be met by obstinacy, and, if the figurative expression may be allowed, a "tug of war" becomes the order of affairs. In addition to the exercises it is advisable to let the child wear a light instrument which shall, when the foot is used, prevent the stronger muscles completing their full degree of contraction. The use of leg instruments is a method of treatment that does not commend itself to some surgeons; the conventional objection to their employment is that it is a wrong principle to require a weak limb to perform an extra task in carrying the weight of the appliance. This was a very fair argument in days gone by, but with the excellent steel now used by skilled mechanicians the weight of an instrument is a very small matter; to be sure, that clumsy contrivance the old "boot and iron" is still a com mercial article, but it is only made and used by those who are not acquainted with the mora recent improvements in orthopædic mechanism. A well-contrived appliance can be obtained which will offer resistance to the pull of any one muscle, or combination of muscles, to the exact degree that the surgeon may deem necessary and will also favour the action of any weak muscle that may need such assistance.
When such remedial measures as those suggested fail to prevent the development of intractility or to overcome the defect when already present, then tenotomy must be resorted to. In the treatment of paralytic talipes the employment of tenotomy requires the exercise of considerable discretion on the part of the surgeon; the operation is capable not only of relieving intractility but also of producing a reverse effect in rendering the muscular fibres too lax. Upon its introduction and for a long time subsequent thereto tenotomy was practised with the object of lengthening the tendon of an abnormal muscle-." contracted tendons" as they were called in those dnys-and much discussion took place as to the correct method of treating a tendon after section. One set of authorities favoured the immediate separation of the cut ends so that a new patch might be added of exactly the required length other practitioners preferred to bring the newly divided surfaces into apposition and afterwards gradually to obtain elongation of the reparative tissue as the healing process advanced, but both parties disregarded the part played by the muscular fibres after their tendinous attachment had been severed. Even now, when it has been recognised that a disorganised muscle and not a contracted tendon is the structure requiring correction no heed is paid to the changes in the muscle and no distinction is made as to the behaviour of the shortened fibres in cases of talipes of different origin. Now whatever may be the effect of tenotomy in the treatment of cases of congenital, spastic, traumatic, or other origin experience has taught me that with paralytic deformity it is chiefly in the muscular fibres that changes occur after the operation. I have pointed out the remarkable relaxation which intractile fibres undergo when placed in a state of rest and the observance of such result convinces me that precisely the same process occurs after tenotomy which places the muscle in a state of absolute repose. It is quite possible by the use of a Scarpa's shoe or other mechanical means to fix the foot so firmly that it cannot be moved upon the leg and yet fail to obtain entire quietude of the muscles; tenotomy gives this result by throwing the muscle completely out of action in separating it from its attachment.

The management of a case after section of a tendon must be con ructed according to the degree of intractility existing in the fibres of the muscle. This it is which necessitates the exercise of much care because there is a difficulty in ascertaining what may be the exact state of the shortened fibres. One precaution which should always be taken is to give the muscle a period of rest before operating, the length of time advisable being determined by the relative condition of the opposing muscles. When the difference in functional activity is slight a rest for 24 hours will suffice and the greater the preponderance of power possessed by the intractile muscle the more prolonged should be the period of inaction. With the most severe disturbance a week spent in this manner is not too long, for as we have seen in the case of equinus illustrated by Fig. 1 an even longer period will often be desirable. This it must be understood applies only to those muscles which have an important part to play in the movement of the foot; the combined extensors and the tibialis anticus are especially to be noted in this respect. Fortunately, severe intractility in these muscles is nearly always accompanied by other structural defect-contraction of the plantar fascia, for instance, which requires correcting before the time for tenotomy is reached. The period spent in the treatment of the first stage gives the necessary rest required for estimating the exact state of the affected muscle. The best safeguard against the mistake of exceeding the correct degree of extension is to make a constant practice of not attempting to restore the normal degree of relaxation during the time that the healing of the tendon is in progress. For in-tance, in the case of the exten-ors of the foot, for all practical purposes it is sufficient to obtain a degree of flexion that will permit of the knee being brought so far forward over the foot as to be in a line with the end of the great toe. Should there be any further relaxation of the gastrocnemius and soleus muscles after the healing process is completed the adoption of the limit suggested will allow for the additional lengthening, but lacking the observance of such precaution the muscles will be weakened and a case of equinus be converted into one of calcaneus. This unfortunate result, by no means of uncommon occurrence, of course places the foot in much worse condition than when in its first state of deformity.

It is with conditions of disturbed muscular balance in 
which the difference is but slightly in favour of one set of opposing muscles that the greater nicety is required in employing tenotomy as the means of ccrrecting the defect, cases in which the extensor muscles have become shortened and limit the flexion movement of the foot to within a degree or so of a right-angled position on the leg, of which an example is given in Fig. 4. This condition, which is very

\section{FrG. 4.}

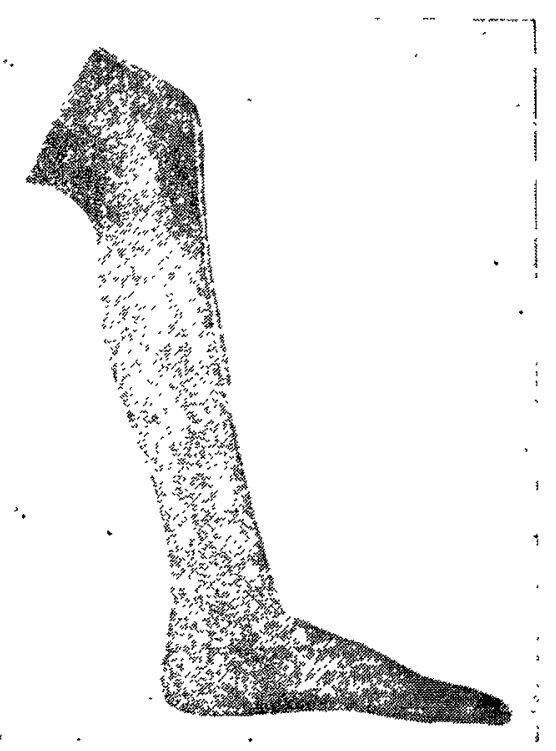

frequently the result of poliomyelitis, is commonly described as "right-angled contraction of the tendo Achillis" (a term introduced in the early days of orthopædic study and retained as a matter of convenience); it not only seriously interferes with the walkirg power of the subject but also leads to the development of several varieties of structural change in the foot. It should always be corrected by tenotomy, other measures having failed, and there need be no anxiety as to the success of the operation if a little care is exercised during the period that the tendon is reuniting. Immediately on section of the tendon the foot must be placed in a position of full extension on the leg so as to bring the points of attachment of the muscles as close together as possible and this position should be maintained for from seven to ten days. The foot may then during the

Fig. 5.

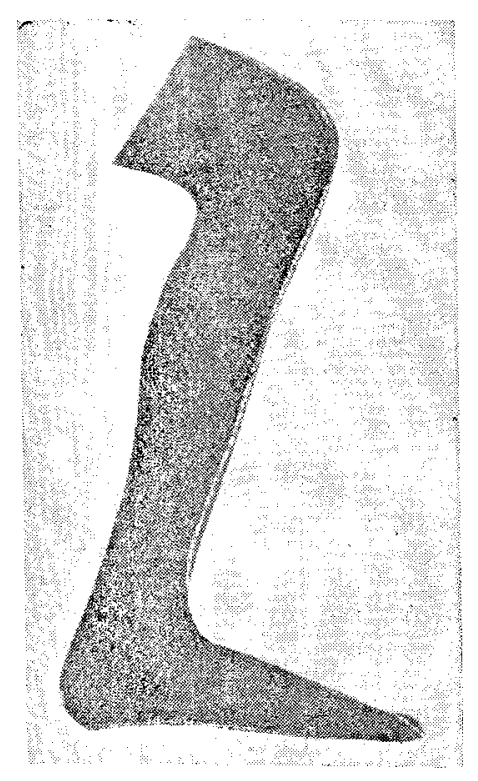

next ten days be gradually placed at a right angle with the leg and on no account should it be flexed beyond this angle nutil the union of the tendon is perfect. The relaxation of the fibres is allowed by this method of procedure to take place spontaneously; they avail themselves of the absolute repose in which they are placed to assume their normal degree of length.

To those surgeons who may see danger in the use of tenotomy in such cases, however carefully conducted, a method of operating which I have successfully employed may be recommended as a perfectly safe course to adopt. This consists in making a partial instead of a complete section of the tendon of the intractile muscle. The case, illustrated by Fig. 4, was thus treated and the result is shown in Fig. 5 ; the degree of flexion obtained might with advantage have been increased; nevertheless, the improvement is well-marked and the patient derived great benefit from the added morement at the ankle-joint. I hare subjected six cases of a similar nature to the same operation and in those which I have been able to observe the subsequent behaviour of the muscles there has been no return of intractility. The tendo Achillis was cut to a depth which left about one-fourth of its bulk undivided, the anterior three-fourths being severed and the posterior fourth left intact. The attempt to replace the foot was commenced on the fifth day after operation and proceeded with as rapidly as rossible; no force was used but flexion was steadily obtained by the use of a Scarpa's shoe. I was led to making the experiment of partial division in such cases from an experience with an Achilles tendon which, owing to frequent attacks of cellulitis, had become adberert to the skin, the skin itself being so thin and ill-nourished that it was impossible to separate it from the tendon. An attempt was made to perform complete tenotomy at the urgent request of the patient who suffered much from the state of his foot, but a few of the posterior fibres were of necessity left undivided. There was sufficient attachment of the muscle remaining to give reason for suspecting that the operation would cause no subsidence of intractility, an unfavourable anticipation which was not fulfilled, as the muscle relaxed to a $d_{t}$ gree which afforded sufficient flexion movement at the ankle. It is difficult to account for the effect produced by the semi-division of a tendon, but there can be no doubt that the operation is capable of affording relief where ordinary measures of treatment have failed. It occurred to me that the relaxing of the fibres might simply be due to prolongation of the period of rest and cases were treated to test this point; some weeks of confinement of the foot in a Scarpa's shoe were given before the operation was employed; in every case immediate improvement followed upon the partial division.

Wimpole-street, $\mathrm{W}$.

\section{TWO CASES OF GLANDERS.}

By E. W. GOODALL, M.D. Lond.,

MEDICAL SUPERINTENDENT, EASTERN FEVER HOSPITAL, HOMERTON, N.E.

CASE 1.-A man, aged 55 years, was admitted into the Eastern Fever Hospital on Oct. 28th, 1903. He was a printer by occupation. The history of his illness as given by his wife was that he had been seized with shivering and pains in the limbs on Oct. 9th. On the 11th he was worse and had to stay in bed, having febrile symptoms. As he did not improve under medical treatment at home he was taken on the 20th to the out-patient department of a general hospital whence he was sent to the Eastern Hospital as a case of typhoid fever.

On admission the patient was found to be very ill. $\mathrm{He}$ was in a semi-comatose state with muttering delirium. The temperature was $102 \cdot 4^{\circ} \mathrm{F}$, the pulse was 112 , and the respirations were 48 . On the skin were to be seen a number of small superficial pustules; they were most abundant on the chest, the outer side of the right leg, and on the right forearm. There were a few on the left cheek, the back, the outer side of the left thigh, and the left unper arm. Besides these pustules there were several swellings of varying size (from one to three inches in diameter) in different places; there was one on the right frontal eminence; there were two on the right forearm, two on the left forearm, one on the right leg, and one on the left leg. The largest swellings fluctuated, the smaller ones did not, but felt nodular. These were freely moveable; some appeared to be in the subcutaneous tissue, others were dintinctly in muscles. The largest swelling was that on the right leg. None of the swellings were connected with bone. The tongue was covered with moist white fur. The fauces were normal ; there was no discharge from the nose. The urine contained a cloud of albumin. The heart was enlarged. The lungs were slightly emphysematous; there was á friction 